\title{
Deep ALMA imaging of the merger NGC 1614 Is $\mathrm{CO}$ tracing a massive inflow of non-starforming gas?
}

\author{
S. König ${ }^{1}$, S. Aalto ${ }^{1}$, S. Muller ${ }^{1}$, J. S. Gallagher $\mathrm{III}^{2,1}$, R. J. Beswick ${ }^{3}$, C. K. Xü ${ }^{4}$, and A. Evans ${ }^{5,6}$ \\ ${ }^{1}$ Chalmers University of Technology, Department of Earth and Space Sciences, Onsala Space Observatory, 43992 Onsala, Sweden \\ e-mail: sabine.koenig@chalmers.se \\ 2 Department of Astronomy, University of Wisconsin, 475 N. Charter Street, Madison, WI, 53706, USA \\ 3 University of Manchester, Jodrell Bank Centre for Astrophysics, Oxford Road, Manchester, M13 9PL, UK \\ ${ }^{4}$ Infrared Processing and Analysis Center, MS 100-22, California Institute of Technology, Pasadena, CA, 91125, USA \\ 5 NRAO, 520 Edgemont Road, Charlottesville, VA, 22903, USA \\ ${ }^{6}$ University of Virginia, Charlottesville, VA, 22904, USA
}

Received 16 March 2016 / Accepted 7 July 2016

\begin{abstract}
Aims. Observations of the molecular gas over scales of $\sim 0.5$ to several kpc provide crucial information on how molecular gas moves through galaxies, especially in mergers and interacting systems, where it ultimately reaches the galaxy center, accumulates, and feeds nuclear activity. Studying the processes involved in the gas transport is one of the important steps forward to understand galaxy evolution.

Methods. ${ }^{12} \mathrm{CO},{ }^{13} \mathrm{CO}$, and $\mathrm{C}^{18} \mathrm{O} 1-0$ high-sensitivity ALMA observations $\left(\sim 4^{\prime \prime} \times 2^{\prime \prime}\right)$ were used to assess the properties of the largescale molecular gas reservoir and its connection to the circumnuclear molecular ring in the merger NGC 1614. Specifically, the role of excitation and abundances were studied in this context. We also observed the molecular gas high-density tracers CN and CS.

Results. The spatial distributions of the detected ${ }^{12} \mathrm{CO} 1-0$ and ${ }^{13} \mathrm{CO} 1-0$ emission show significant differences. ${ }^{12} \mathrm{CO}$ traces the large-scale molecular gas reservoir, which is associated with a dust lane that harbors infalling gas, and extends into the southern tidal tails. ${ }^{13} \mathrm{CO}$ emission is for the first time detected in the large-scale dust lane. In contrast to ${ }^{12} \mathrm{CO}$, its line emission peaks between the dust lane and the circumnuclear molecular ring. $\mathrm{A}^{12} \mathrm{CO}-{ }^{-t o}-{ }^{13} \mathrm{CO} 1-0$ intensity ratio map shows high values in the ring region $(\sim 30)$ that are typical for the centers of luminous galaxy mergers and even more extreme values in the dust lane (>45). Surprisingly, we do not detect $\mathrm{C}^{18} \mathrm{O}$ emission in NGC 1614 , but we do observe gas emitting the high-density tracers $\mathrm{CN}$ and $\mathrm{CS}$.

Conclusions. We find that the ${ }^{12} \mathrm{CO}-\mathrm{to}_{-}{ }^{13} \mathrm{CO} 1-0$ line ratio in NGC 1614 changes from $>45$ in the $2 \mathrm{kpc}$ dust lane to 30 in the starburst nucleus. This drop in ratio with decreasing radius is consistent with the molecular gas in the dust lane being kept in a diffuse, unbound state while it is being funneled toward the nucleus. This also explains why there are no (or very faint) signs of star formation in the dust lane, despite its high ${ }^{12} \mathrm{CO}$ luminosity. In the inner $1.5 \mathrm{kpc}$, the gas is compressed into denser and most likely self-gravitating clouds (traced by $\mathrm{CN}$ and $\mathrm{CS}$ emission), allowing it to power the intense central starburst. We find a high ${ }^{16} \mathrm{O}$-to${ }^{18} \mathrm{O}$ abundance ratio in the starburst region $(\geq 900)$, typical of quiescent disk gas. This is surprising because by now, the starburst is expected to have enriched the nuclear interstellar medium in ${ }^{18} \mathrm{O}$ relative to ${ }^{16} \mathrm{O}$. We suggest that the massive inflow of gas may be partially responsible for the low ${ }^{18} \mathrm{O} /{ }^{16} \mathrm{O}$ abundance since it will dilute the starburst enrichment with unprocessed gas from greater radial distances. The ${ }^{12} \mathrm{CO}$-to- ${ }^{13} \mathrm{CO}$ abundance of $>90$ we infer from the line ratio is consistent with this scenario. It suggests that the nucleus of NGC 1614 is in a transient phase of its evolution where the starburst and the nuclear growth is still being fuelled by returning gas from the minor merger event.
\end{abstract}

Key words. galaxies: evolution - galaxies: individual: NGC 1614 - galaxies: starburst - galaxies: active - radio lines: galaxies ISM: molecules

\section{Introduction}

Minor mergers, which are close galaxy interactions where the partners have an unequal mass ratio, constitute the majority of interacting events in the Universe. The gas accretion and nuclear feeding mechanisms of these mergers are different from major mergers (equal-mass interactions, e.g., Naab et al. 2009). Minor mergers are important for our understanding of galaxy evolution. In particular, they provide insights into how high-redshift spheroidal galaxies evolve into systems at low-redshift, such as local elliptical galaxies, and the formation of galaxy bulges (e.g., Naab et al. 2009; Weinzirl et al. 2009). In minor mergers, gas introduced into the interacting system by the disturbing companion can generally be found at large radii from the center of the merger remnant (Bournaud et al. 2005). Stellar bars, causing gravitational torques to affect the gas, and/or tidal torques invoked by the disturbing companion, may lead to the transport of the molecular gas along the large-scale dust lanes to the mergers center, with this material acting to fuel both star formation and nuclear accretion (e.g., Simkin et al. 1980; Scoville et al. 1985; Jogee 2006; Weinzirl et al. 2009). There the gas may form polar rings that can appear as dust lanes when seen edge-on (e.g., Combes 1988; Shlosman et al. 1989). We have found evidence that gas is funneled along polar rings (Aalto \& Hüttemeister 2000; Aalto et al. 2010; König et al. 2013, 2014). Tracing this gas is vital to understand the underlying galaxy-evolution and star-formation mechanisms in this class of mergers. 
Spectral lines of molecular isotopologs (isotopic variants) can be used to study the effect of infall, enrichment and gas physical conditions. Elevated line ratios between the ${ }^{12} \mathrm{C}$ and ${ }^{13} \mathrm{C}$ variants of $\mathrm{CO}$ have, for example, been proposed to indicate a replenishment of relatively unprocessed disk gas (e.g., Casoli et al. 1992; Henkel \& Mauersberger 1993; Taniguchi et al. 1999). However, the ratio cannot be used to infer values on ${ }^{12} \mathrm{C} /{ }^{13} \mathrm{C}$ without taking effects of optical depth and line excitation (temperature, line width) into account. For example, ${ }^{12} \mathrm{CO} /{ }^{13} \mathrm{CO}$ line ratios may be low in quiescent disk gas and higher in hot nuclear gas without a change in ${ }^{12} \mathrm{C} /{ }^{13} \mathrm{C}$ abundances, or even with the opposite abundance gradient (e.g., Aalto et al. 1995, 1997; Davis et al. 2015). Dynamical effects are also found to have a strong influence on the observed ${ }^{12} \mathrm{CO} /{ }^{13} \mathrm{CO}$ line ratios (e.g., Tosaki et al. 2002; Meier \& Turner 2004; Aalto et al. 2010). In addition, issues of selective photo destruction also need to be addressed (e.g., Aalto et al. 1995).

Isotopic variants of oxygen, ${ }^{16} \mathrm{O}$ and ${ }^{18} \mathrm{O}$, are used to trace enrichment by massive stars (e.g., Henkel \& Mauersberger 1993; González-Alfonso et al. 2014; Falstad et al. 2015). González-Alfonso et al., for example, found very high ${ }^{18} \mathrm{O}$ abundances in the nearby ULIRG quasar Mrk 231 which they attributed to an evolved nuclear starburst.

NGC $1614\left(D=64 \mathrm{Mpc}, 1^{\prime \prime}=310 \mathrm{pc}, L_{8-1000 \mu \mathrm{m}} \sim 4 \times\right.$ $10^{11} L_{\odot}$, Sanders et al. 2003) is a minor merger that shows intense nuclear activity and a complex morphology, with a minor axis dust lane crossing the optical body close to the nucleus. The presence of a circumnuclear ring $(r \sim 300 \mathrm{pc})$ has been reported from different tracers, for example $\mathrm{Pa} \alpha$ (Alonso-Herrero et al. 2001), PAH (Väisänen et al. 2012), multiple transitions of $\mathrm{CO}$ at $\mathrm{mm}$ wavelengths (König et al. 2013; Sliwa et al. 2014; Xu et al. 2015), and in the radio continuum (e.g., Olsson et al. 2010; König et al. 2013). Most of the nuclear activity originates from a very young starburst residing in the ring itself (6-7 Myr, Puxley \& Brand 1999; Kotilainen et al. 2001; Schwartz \& Martin 2004) and an older starburst at its center (>10 Myr, Alonso-Herrero et al. 2001). Two competing formation scenarios for the ring have been proposed: a "wildfire"scenario (Alonso-Herrero et al. 2001) where the ring is the result of an outward propagating starburst, or that the ring is formed at the location of a Lindblad resonance and is fueled by gas moving in along the dust lanes onto the ring (König et al. 2013).

A number of emission lines tracing different physical conditions originate from the molecular gas in NGC 1614. A considerable fraction of the total molecular gas resides in the central dust lanes but is not involved in the nuclear activity $\left({ }^{12} \mathrm{CO} 1-0\right.$, Olsson et al. 2010; Sliwa et al. 2014; García-Burillo et al. 2015). Further studies of the ${ }^{12} \mathrm{CO} 1-0$ have revealed a molecular outflow in NGC 1614 (García-Burillo et al. 2015).

In our previous high-resolution CO studies of NGC 1614 we not only discovered molecular gas in the starburst ring traced by $\mathrm{CO} 2-1$, but also that the majority of the $\mathrm{CO}$ emission of NGC 1614 is located in the dust lane and is apparently not starforming. We suggested that the gas here may be infalling and in a diffuse, unbound state.

To investigate the nature of the large-scale molecular gas of the nearby merger NGC 1614 we obtained high-sensitivity ALMA ${ }^{12} \mathrm{CO},{ }^{13} \mathrm{CO}$, and $\mathrm{C}^{18} \mathrm{O} 1-0$ data. We also detected CN 1-0 and CS 2-1.

In this paper we present the results of a study of the properties of the molecular gas within a radius of $\sim 3 \mathrm{kpc}$ in NGC 1614. An analysis of our higher angular resolution ALMA data will be presented in a later paper. Throughout the paper, we are concerned with pure rotational transitions of the observed molecules between upper state $J^{\prime}=j$ and lower state $J=i$ that are labeled $j-i$. Section 2 describes the observations and the data analysis, in Sect. 3 the results of the observations are presented, and we discuss their implications in Sect. 4.

\section{Observations, data reduction and analysis}

NGC 1614 was observed with the Atacama Large Millimeter/submillimeter Array (ALMA) in band $3(3 \mathrm{~mm})$ as part of Cycle 2 observations of project 2013.1.00991.S (PI: S. König). Here we focus on the data obtained with the ALMA Compact Array (ACA) and the ALMA main array in compact configuration. The ACA observations took place on 2014 June 16 and 30, the ALMA compact configuration observations on 2014 December 4 and 5 . The phase center of the observations was located at $\alpha=04: 34: 00.02688$ and $\delta=-08.34 .44 .5692$ (J2000). Two tunings were obtained centered on $113.5 \mathrm{GHz}$ and $108.5 \mathrm{GHz}$, where the redshifted frequencies of the ${ }^{12} \mathrm{CO} 1-0$ and ${ }^{13} \mathrm{CO} 1-0$ lines are located. Other lines observed within the ALMA band are $\mathrm{C}^{18} \mathrm{O} 1-0, \mathrm{CN} 1-0$ and $\mathrm{CS} 2-1$. The $3 \mathrm{~mm}$ continuum was reconstructed using line-free channels. We used four spectral windows of $1.875 \mathrm{GHz}$ bandwidth $\left(\sim 5000 \mathrm{~km} \mathrm{~s}^{-1}\right)$ each, with a velocity resolution of $\sim 5 \mathrm{~km} \mathrm{~s}^{-1}$, after Hanning smoothing. During the observations, different sources were observed for calibration purposes: Uranus as flux calibrator, J0339-0146 as bandpass calibrator, and J0423-0120 as bandpass and phase calibrator. With baseline lengths of between $8.8 \mathrm{~m}$ and $49.0 \mathrm{~m}$, the ACA data are sensitive to scales smaller than $\sim 38^{\prime \prime}$. The compact configuration main array ALMA data are sensitive to scales smaller than $\sim 22^{\prime \prime}$ (baselines: $15.0 \mathrm{~m}-390 \mathrm{~m}$ ).

For all emission lines, sets of visibilities from ACA and the compact configuration observations were combined by weighting the individual data sets in the $u v$ plane to guarantee a continuous amplitude vs. $u v$-distance distribution. The combined data set was then deconvolved using the "clean" task in CASA, and individual data cubes were created for each observable (continuum and emission lines). The resulting beam sizes and sensitivities are shown in Table 1.

After calibration and imaging within $\mathrm{CASA}^{1}$ (McMullin et al. 2007), both visibility sets were converted into FITS format and imported in GILDAS/MAPPING ${ }^{2}$ for further analysis. All integrated intensity maps in this paper are moment-zero maps. To obtain the ${ }^{12} \mathrm{CO}-$ to- $^{13} \mathrm{CO} 1-0$ map in Fig. 6 we used the integrated intensity maps of the two emission line distributions without a-priori clipping. The resulting maps were smoothed to a common beam at $5^{\prime \prime} \times 5^{\prime \prime}$, and then the ratio map was obtained on a pixel-by-pixel basis. Only pixels with signal-to-noise ratios equal to or higher than 5 in the ratio map were taken into account and are depicted in the resulting image.

\section{Results}

\section{1. $3 \mathrm{~mm}$ continuum}

The $3 \mathrm{~mm}$ continuum (Fig. 1) is centrally peaked at the nucleus of NGC 1614. The total integrated flux recovered from the area enclosed by the $3 \sigma$ contours in the uniformly weighted map is $\sim 12.9 \mathrm{mJy}$. The size and structure of the distribution, however, show more extended features as well. The emission seems to be elongated with a northeast-southwest direction similar to

\footnotetext{
http://casa.nrao.edu/

http: //wWw . iram. fr/IRAMFR/GILDAS
} 
Table 1. Properties of the observed lines and resulting images.

\begin{tabular}{|c|c|c|c|c|c|c|}
\hline Observable & $\begin{array}{c}\text { Observing frequency } \\
{[\mathrm{GHz}]}\end{array}$ & Beam & $\begin{array}{c}\text { Channel width } \\
{\left[\mathrm{km} \mathrm{s}^{-1}\right]}\end{array}$ & $\begin{array}{c}\text { Sensitivity } \\
{\left[\mathrm{mJy}^{-1} \text { beam }^{-1}\right]}\end{array}$ & Integrated intensity & $\begin{array}{l}\text { Peak flux } \\
{[\mathrm{Jy}]}\end{array}$ \\
\hline continuum & 105.0 & $4{ }^{\prime \prime} 46 \times 1^{\prime \prime} .68$ & - & 0.06 & $12.9 \pm 0.8 \mathrm{mJy}$ & - \\
\hline${ }^{12} \mathrm{CO} 1-0$ & 113.5 & $4{ }^{\prime \prime} 38 \times 11^{\prime \prime} .84$ & 10 & 1.6 & $241 \pm 1.0 \mathrm{Jy} \mathrm{km} \mathrm{s}^{-1}$ & $1.1 \pm 0.02$ \\
\hline $\mathrm{CN} 1-0 \mathrm{~J}=3 / 2-1 / 2$ & 111.4 & $4{ }^{\prime \prime} 06 \times 1^{\prime \prime} .61$ & 10 & 1.3 & $3.8 \pm 1.0 \mathrm{Jy} \mathrm{km} \mathrm{s}^{-1}$ & $0.017 \pm 0.003$ \\
\hline $\mathrm{CN} 1-0 \mathrm{~J}=1 / 2-1 / 2$ & 111.7 & $4{ }^{\prime \prime} 06 \times 11^{\prime \prime} 61$ & 10 & 1.3 & $7.2 \pm 1.0 \mathrm{Jy} \mathrm{km} \mathrm{s}^{-1}$ & $0.027 \pm 0.003$ \\
\hline${ }^{13} \mathrm{CO} 1-0$ & $-\overline{108.5}$ & $44^{\prime \prime} 18 \times 22^{\prime \prime} \cdot 24$ & $-\overline{10}$ & $--\overline{1.4}$ & $6.6 \pm 1.0 \mathrm{Jy} \mathrm{km} \mathrm{s}-1$ & $\overline{0.04 \pm 0.004}$ \\
\hline $\mathrm{C}^{18} \mathrm{O} 1-0$ & 108.1 & $4.20 \times 2.25$ & 60 & 0.8 & $<0.1 \mathrm{Jy} \mathrm{km} \mathrm{s}^{-1}$ & - \\
\hline CS 2-1 & 96.4 & $4^{\prime \prime} .65 \times 22^{\prime \prime} 55$ & 30 & 0.8 & $1.3 \pm 1.0 \mathrm{Jy} \mathrm{km} \mathrm{s}^{-1}$ & $0.008 \pm 0.001$ \\
\hline
\end{tabular}

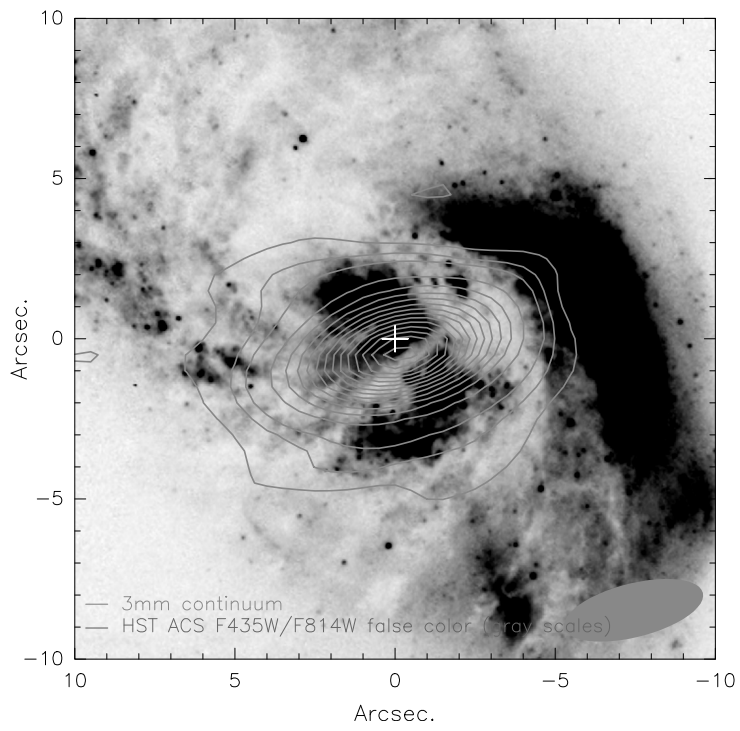

Fig. 1. $3 \mathrm{~mm}$ continuum emission (in gray contours) on top of an HST ACS $F 435 W$ filter gray-scale image to facilitate a comparison of the location and distribution of the emission with respect to the dust lanes at the center of NGC 1614. This image of the $3 \mathrm{~mm}$ continuum emission was created using uniform weighting, which resulted in a sensitivity of $1 \sigma=59.8 \mu \mathrm{Jy}$ beam $^{-1}$. The contours start at $3 \sigma$ and are spaced in steps of $5 \sigma$. The cross marks the phase center of the observations, placed at the center of the molecular gas ring. The beam (see also Table 1) is shown in the lower right corner. North is up, east to the left.

what was observed for ${ }^{12} \mathrm{CO} 1-0$ previously (Olsson et al. 2010; Sliwa et al. 2014). The continuum emission is clearly associated with the bulk of the ${ }^{12} \mathrm{CO}$ emission, but also with the dust lanes in the center of NGC 1614. Previously published observations of the $1.3 \mathrm{~mm}$ continuum (Wilson et al. 2008; König et al. 2013) show a more compact distribution $\left(\sim 6-7^{\prime \prime}\right)$ of the emission, even with comparable spatial resolution. This might be partially due to the excellent continuum sensitivity in our observations compared to previous data sets, but it could also be that the continuum at higher frequencies is more associated with the gas in the molecular ring (König et al. 2013) than the larger-scale molecular gas reservoir (Olsson et al. 2010; Sliwa et al. 2014).

\section{2. ${ }^{12} \mathrm{CO} 1-0$}

The ${ }^{12} \mathrm{CO} 1-0$ emission in NGC 1614 is more extended than the $3 \mathrm{~mm}$ continuum emission. In addition to the difference in size, the ${ }^{12} \mathrm{CO}$ distribution also shows a significantly different structure than the $3 \mathrm{~mm}$ continuum (Fig. 2). Location and general morphology are in agreement with the results of Olsson et al. (2010), Sliwa et al. (2014), and García-Burillo et al. (2015): the gas distribution appears centrally peaked with a significant elongation along a northeastsouthwest direction, associated with the dust lanes in the center of NGC 1614. Olsson et al. (2010) found an extent of about 11", Sliwa et al. (2014) about 14", García-Burillo et al. (2015) about $12^{\prime \prime}$. In our data the extension is roughly $25^{\prime \prime}$ in the northeastsouthwest direction. This difference is due to the higher sensitivity and the integration of short-spacing observations in our data set. Additionally, the distribution in Fig. 2 shows features in the molecular gas that were previously unknown. The extension towards the south, for example, consists of low-surface brightness gas that extends into the tidal tails to the south-southwest of the main body of the galaxy. Hints for the feature extending toward the southeast, continuing in the direction of the minor nuclear dust lane, can already be detected in Sliwa et al. (2014) and García-Burillo et al. (2015). Our higher-sensitivity, improved $u v$-coverage observations now clearly confirm their presence. The total interferometric integrated flux enclosed in the $3 \sigma$ contours is $\sim 241 \mathrm{Jy} \mathrm{km} \mathrm{s}^{-1}$, which is about a factor of seven times more than in previous observations of Olsson et al. (2010), and a factor of two more than previously observed by Scoville et al. (1989). A comparison to single-dish observations shows that we recover approximately $115 \%$ of the flux that has recently been detected by Costagliola et al. (2011).

The spectrum of the ${ }^{12} \mathrm{CO} 1-0$ emission (Fig. 2a) shows a double-peaked Gaussian with a FWHM line width of $\sim 250 \mathrm{~km} \mathrm{~s}^{-1}$. The peak flux is at about $1.1 \mathrm{Jy}$.

\section{3. ${ }^{13} \mathrm{CO} 1-0$}

Figure 3 shows the spectrum and integrated intensity distribution of the ${ }^{13} \mathrm{CO} 1-0$ emission in NGC 1614. The emission line appears to be double peaked, with its highest flux being $\sim 0.04 \mathrm{Jy}$, and an integrated flux of $\sim 6.6 \mathrm{Jy} \mathrm{km} \mathrm{s}^{-1}$ at a line width (FWHM) of about $250 \mathrm{~km} \mathrm{~s}^{-1}$. The recovered flux corresponds to $98 \%$ of the total flux detected with single-dish observations (Costagliola et al. 2011). Compared to previous interferometric observations, the sensitivity in our observations is increased by a factor of seven. As a result we recover an integrated flux that is twice as high as in these data (Sliwa et al. 2014). The integrated intensity distribution shows the ${ }^{13} \mathrm{CO} 1-0$ emission to be situated in an elongated structure of roughly $12^{\prime \prime}$ extending from the northeast of the nucleus of NGC 1614 to the southwest of it. The peak of the distribution, however, is located slightly northwest of the nucleus, where the ${ }^{12} \mathrm{CO} 2-1$ emission in the molecular ring connects to the dust lane (Fig. A.1, König et al. 2013). A comparison with an Hubble Space Telescope (HST) image (Fig. 3b) shows that the ${ }^{13} \mathrm{CO}$ emission appears to follow the main high brightness star forming structures at the center of NGC 1614. 

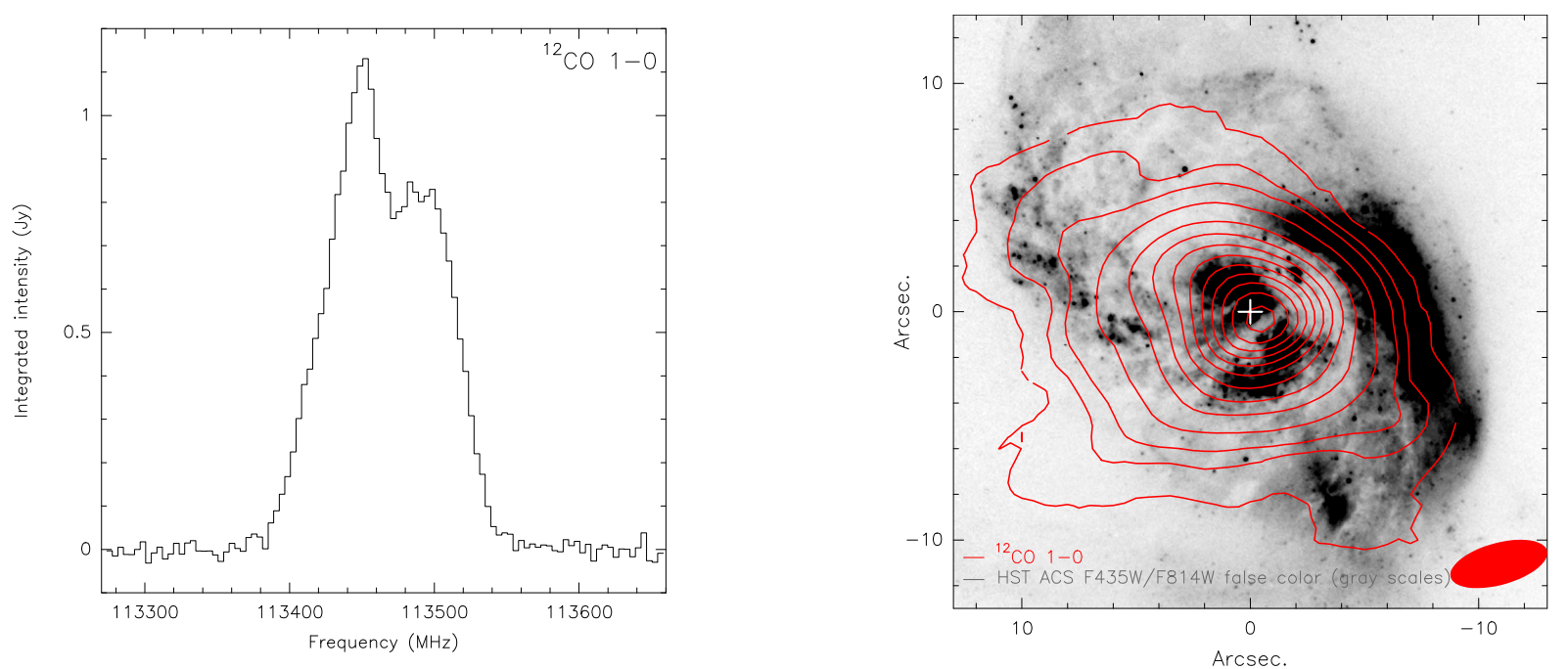

Fig. 2. ${ }^{12} \mathrm{CO} 1-0$ emission in NGC 1614 . The spectrum of the ${ }^{12} \mathrm{CO} 1-0$ emission with a channel width of $10 \mathrm{~km} \mathrm{~s}^{-1}$ is shown on the left, an overlay of the integrated emission contours on top of an HST ACS F435W filter gray-scale image is shown on the right. The integrated intensity map was obtained using uniform weighting. The resulting $1 \sigma$ sensitivity is $\sim 1.6 \mathrm{mJy} \mathrm{beam}^{-1}$, the synthesized beam (see Table 1 ) is depicted in the lower right corner. The first three contour levels are at $3 \sigma, 13 \sigma$, and $23 \sigma$. For legibility purposes, the next contour is at $50 \sigma$; from there on the subsequent contours are spaced by $50 \sigma$ until the peak flux is reached. The cross marks the phase center. North is up, east to the left.
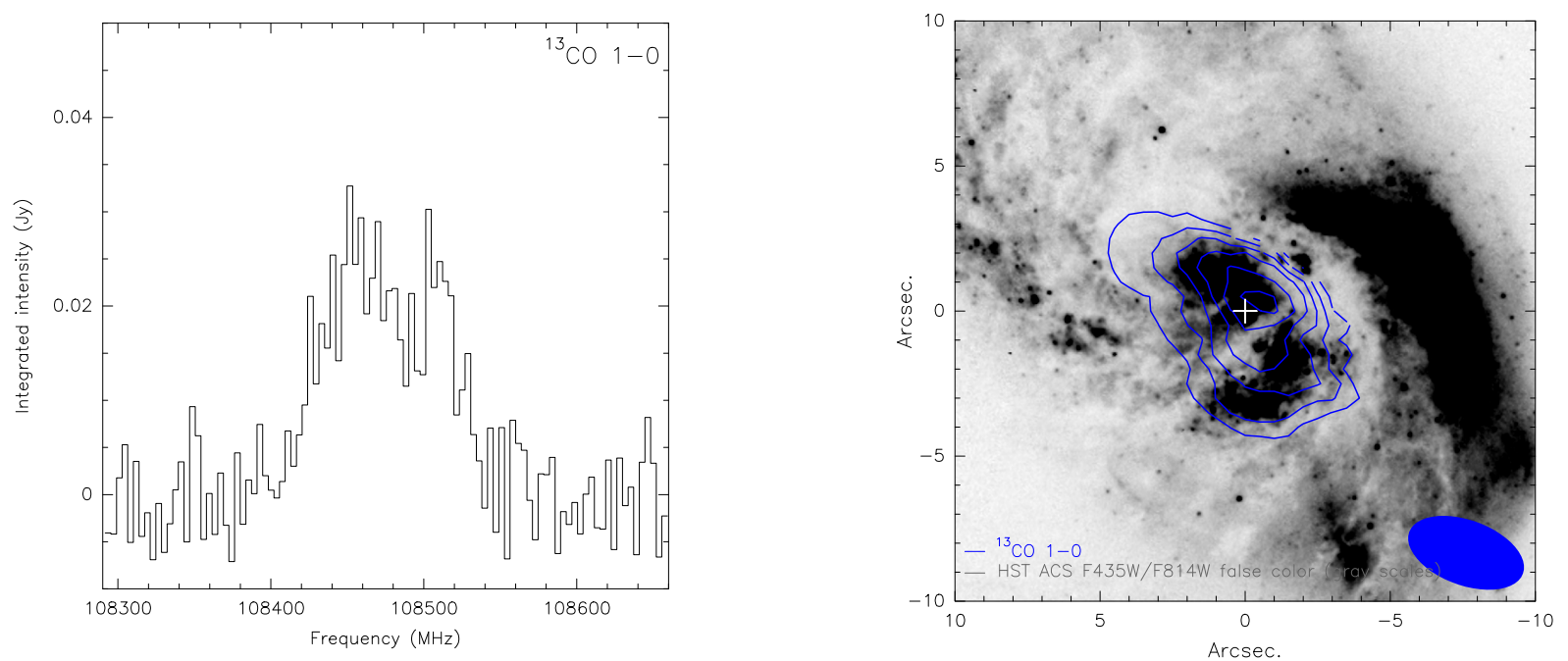

Fig. 3. Spectrum of the ${ }^{13} \mathrm{CO} 1-0$ emission in NGC 1614 (channel width: $10 \mathrm{~km} \mathrm{~s}^{-1}$, left), and overlay of the integrated emission contours on top of an HST ACS $F 435 W$ filter gray-scale image (right). The contours in the map obtained from natural weighting start at $4 \sigma$ and are spaced in steps of $3 \sigma$. The $1 \sigma$ sensitivity here is $\sim 1.4$ mJy beam $^{-1}$. The beam (see Table 1 ) is depicted in the bottom right corner. The cross marks the phase center. North is up, east to the left.

The image furthermore indicates that the extent of the ${ }^{13} \mathrm{CO}$ emission does not cover the complete extent of the western dust lane. Instead, it seems that the bulk of the ${ }^{13} \mathrm{CO} 1-0$ emission is avoiding the dust lane, in contrast to the ${ }^{12} \mathrm{CO} 1-0$ emission (see also Fig. A.2).

\section{4. $\mathrm{C}^{18} \mathrm{O} 1-0, \mathrm{CN} 1-0$, and CS 2-1}

Other molecular emission lines contained in the observed bandwidths are $\mathrm{C}^{18} \mathrm{O} 1-0, \mathrm{CN} 1-0$, and $\mathrm{CS} 2-1$.

The $\mathrm{C}^{18} \mathrm{O} 1-0$ emission line was not detected. An upper limit at $3 \sigma$ amounts to $\sim 0.1 \mathrm{Jy} \mathrm{km} \mathrm{s}^{-1}$ within the central $4^{\prime \prime} \times 4^{\prime \prime}$. This flux limit is a factor of more than 8.5 lower than what has been obtained from previous single-dish observations (Costagliola et al. 2011).

The CN $N=1-0$ emission is located in a compact, unresolved distribution at the nucleus of NGC 1614 (Fig. 4b). With a peak flux of $\sim 0.03 \mathrm{Jy}$ and an integrated flux of $7.2 \mathrm{Jy} \mathrm{km} \mathrm{s}{ }^{-1}$, the $J=3 / 2-1 / 2$ component is stronger than the $J=1 / 2-1 / 2$ component (Fig. 4a; peak flux: $\sim 0.02$ Jy, integrated flux: $\sim 3.8 \mathrm{Jy} \mathrm{km} \mathrm{s}^{-1}$ ). The total integrated flux is $\sim 10.3 \mathrm{Jy} \mathrm{km} \mathrm{s}^{-1}$. The average FWHM line width amounts to $\sim 250 \mathrm{~km} \mathrm{~s}^{-1}$. $\mathcal{R}_{\mathrm{CN}}$, the integrated line ratio between the $\mathrm{CN} N=1-0 \quad J=3 / 2-1 / 2$ and $J=1 / 2-1 / 2$ components amounts to $\sim 2$, which indicates that the gas traced by the CN emission in NGC 1614 is optically thin.

The CS 2-1 emission is clearly detected in NGC 1614 (Fig. 5). Its spatial distribution is compact and centered on the nucleus of the galaxy, following the main high-brightness starforming structures in the HST image (Fig. 5b). The peak flux in the spectrum is at $7.7 \mathrm{mJy}$. The integrated flux is found to be roughly $1.3 \mathrm{Jy} \mathrm{km} \mathrm{s}^{-1}$, at a FWHM line width of $\sim 250 \mathrm{~km} \mathrm{~s}^{-1}$.

\section{5. ${ }^{12} \mathrm{CO}-$ to $^{13} \mathrm{CO} 1-0$ line ratio}

From a detailed comparison of the ${ }^{12} \mathrm{CO}$ and ${ }^{13} \mathrm{CO} 1-0$ emission, the distributions indicate an offset in the emission peak location between the two tracers. The ${ }^{12} \mathrm{CO}$ peaks exactly at the nucleus 

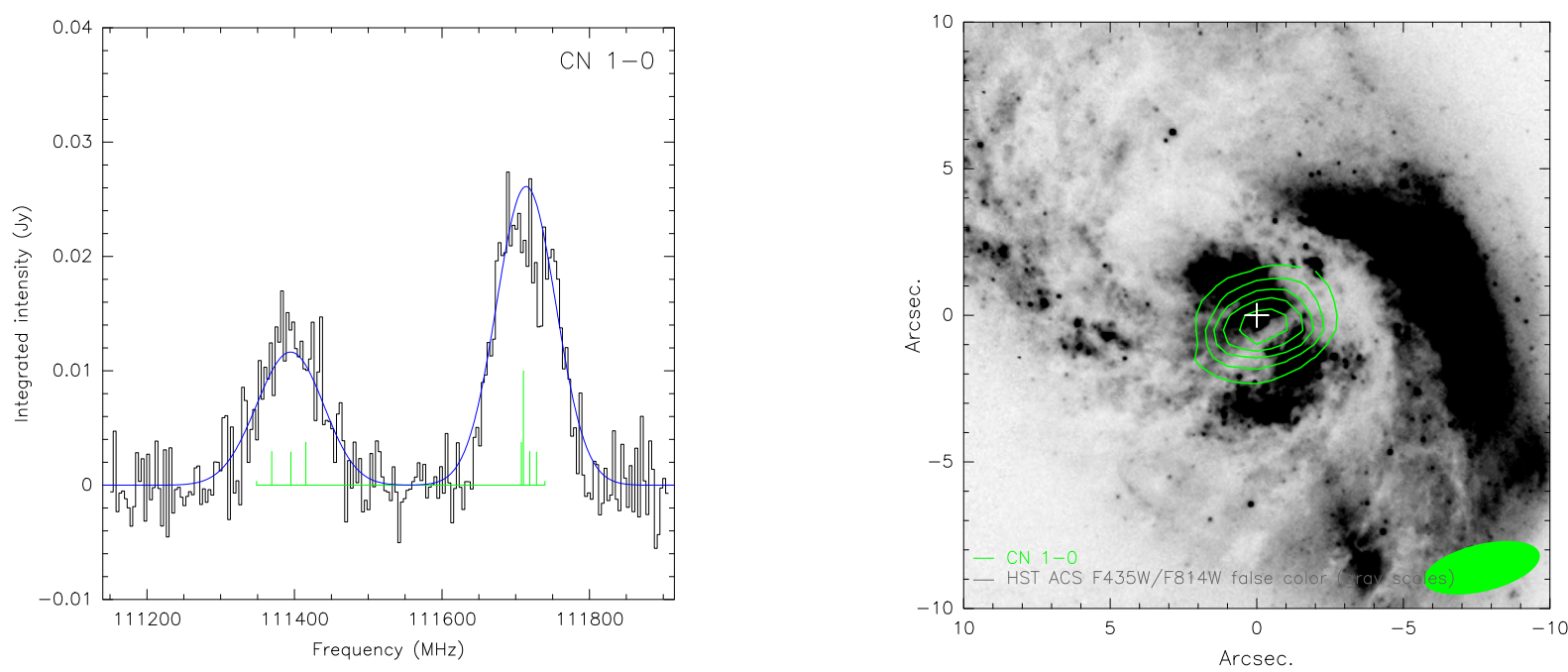

Fig. 4. Spectrum of the CN 1-0 emission in NGC 1614 (left), and overlay of the integrated emission on top of an HST ACS F435W filter grayscale image (right). In the spectrum (channel width: $10 \mathrm{~km} \mathrm{~s}^{-1}$ ) the hyperfine-structure components of CN 1-0 are indicated in green. The blue line represents the convolution of the $\mathrm{CN}$ hyperfine structure with a Gaussian profile. This indicates that the gas traced by the $\mathrm{CN} 1-0$ emission in NGC 1614 is optically thin. The integrated intensity map was obtained using uniform weighting. The resulting sensitivity is $\sim 1.3 \mathrm{mJy} \mathrm{beam}^{-1}$, the resulting beam is depicted in the bottom right corner. The contour levels of the integrated emission start at $5 \sigma$ and are spaced in steps of $5 \sigma$. The beam (see also Table 1) is depicted in the lower right corner. The cross marks the phase center. North is up, east to the left.
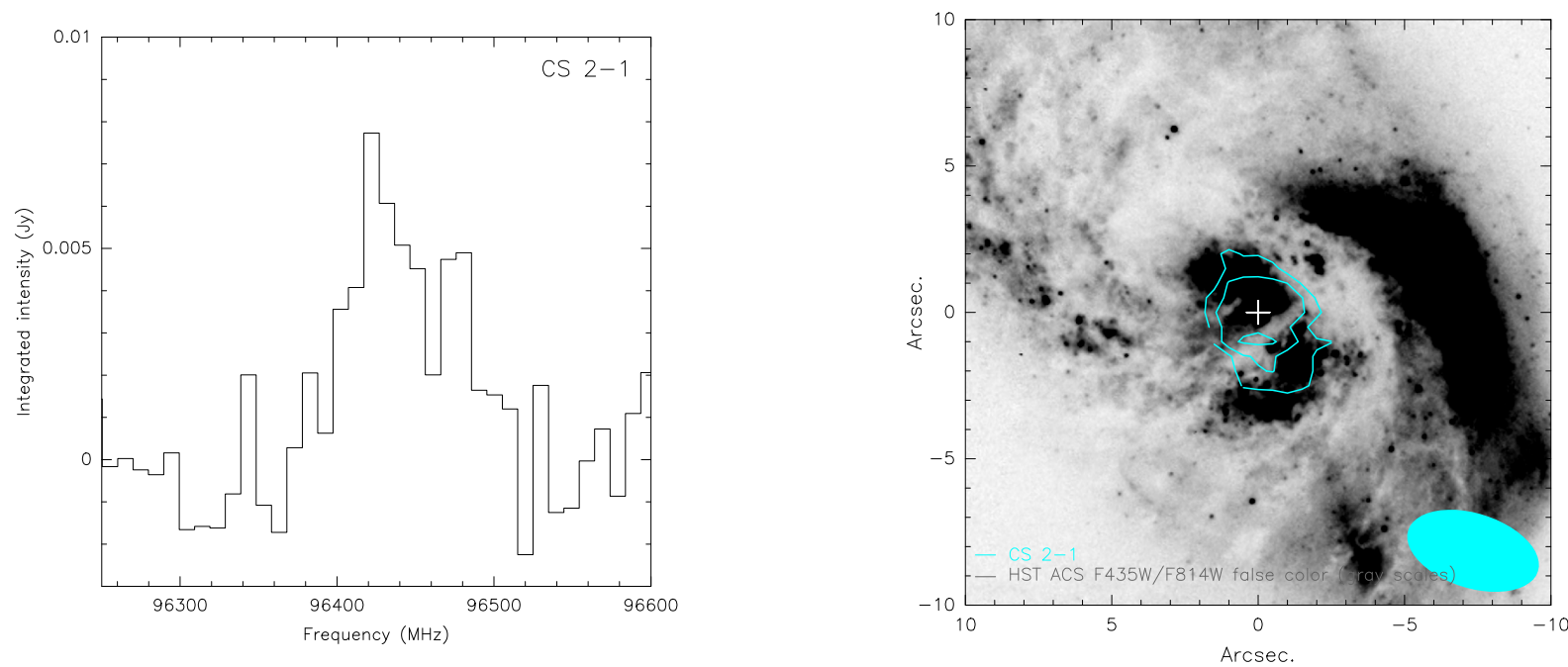

Fig. 5. Spectrum of the CS 2-1 emission in NGC 1614 (left), and overlay of the integrated emission on top of an HST ACS F435W filter gray-scale image (right). The channels in the spectrum were binned to $30 \mathrm{~km} \mathrm{~s}^{-1}$. The contours of the integrated emission in the naturally weighted map start at $3.5 \sigma$ and are spaced in steps of $3 \sigma\left(1 \sigma=0.8 \mathrm{mJy}_{\text {beam }}^{-1}\right)$. The beam (see also Table 1$)$ is depicted. The cross marks the phase center. North is up, east to the left.

of NGC 1614, whereas ${ }^{13} \mathrm{CO}$ peaks slightly to the northwest of it (an overlay image showing the central 14" can be found in the Appendix in Fig. A.2). Furthermore, the two emission distributions are distinctly different in size and structure. A ${ }^{12} \mathrm{CO}-$ to- ${ }^{13} \mathrm{CO} 1-0\left(\mathcal{R}_{10}\right)$ line ratio map (Fig. 6) shows compelling evidence for this. The $\mathcal{R}_{10}$ distribution shows an elongated structure of constant value $(\sim 30)$ that coincides with the location of the minor axis dust lane close to the nucleus (Fig. 6b). Moving along this direction toward the dust lane in the west of the nucleus, $\mathcal{R}_{10}$ increases to its highest values of 40 to 45 at the outer edge of the western dust lane, where the ${ }^{13} \mathrm{CO} 1-0$ is largely absent. Toward the northeastern, eastern, and western edge of the map, the distribution shows more normal values $\left(\mathcal{R}_{10}=10-15\right)$ associated with the gas in the disk of starburst galaxies.

Sliwa et al. (2014) reported $\mathcal{R}_{10}$ similar to our findings. Their values range from about 25 to approximately 40-45. Their line ratio map, however, shows a different distribution compared to our work (Fig. 6) at several locations in NGC 1614: the highest values for $\mathcal{R}_{10}$ are found toward the southern part of the circumnuclear molecular ring. $\mathcal{R}_{10}$ is slightly lower than that to the west (toward the dust lane). The reason might be that Sliwa et al. (2014) only recovered $\sim 50 \%$ of the total flux in their data set, while we essentially recovered $100 \%$ of the single dish flux. The authors found the same lower $\mathcal{R}_{10}$ toward the northeast and east of the ring, however.

Higher resolution observations are necessary to exactly locate the peak in the $\mathcal{R}_{10}$ distribution (König et al., in prep.).

\section{Discussion}

\subsection{Line ratio variations}

For the first time we were able to measure the ${ }^{12} \mathrm{CO}-$ to- $^{13} \mathrm{CO} 1-0$ line ratio in the central dust lanes in NGC 1614 (Sect. 3.5). We report an overall high $\mathcal{R}_{10}$, with increasing values toward the dust western lane. Studies have been published in other nearby minor merger systems, for example, the Medusa merger (Aalto et al. 2010), which show similarly elevated $\mathcal{R}_{10}$. It was suggested that 

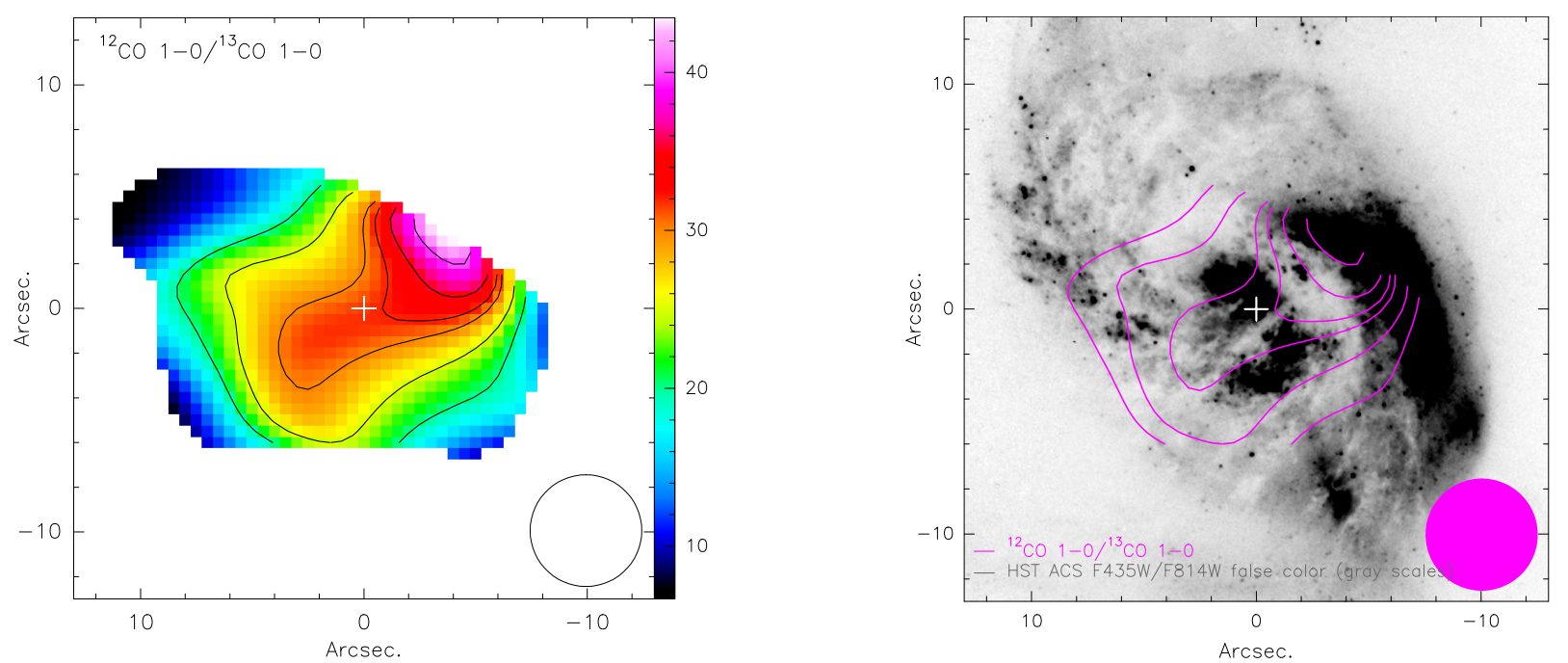

Fig. 6. ${ }^{12} \mathrm{CO}-{ }^{-t o}-{ }^{13} \mathrm{CO}$ integrated intensity line ratio $\left(\mathcal{R}_{10}\right)$ map $($ left $)$ and overlay of the same in contours on top of an HST ACS $F 435 W$ filter gray-scale image (right). The ratio decreases from about 40-45 at the northwestern edge down to $\sim 30$ along the nuclear dust lane, and $\sim 10-20$ at the northeastern, eastern, and western edges. $\mathcal{R}_{10}$ seems to be quite constant along the nuclear dust lane until the ratio steeply increases toward the dust lane in the west. North is up, east to the left, and the beam is shown in the lower right corner of each map. The cross marks the position of the phase center.

the elevated line ratios are the result of two effects, either acting alone or combined: 1) changes in the physical conditions in the gas; or 2) abundance effects (e.g., Meier \& Turner 2004; Aalto et al. 2010). These effects are discussed further below.

\subsubsection{Excitation effects}

\subsubsection{Temperature}

In the context of the discussion in this section, we assume that the abundances of ${ }^{12} \mathrm{CO}$ and ${ }^{13} \mathrm{CO}$ are constant over the central region of interest. High average gas temperatures, low average gas densities, or exceptionally high velocity dispersions in the molecular clouds could be responsible for elevated line ratios (e.g., Aalto et al. 1995; Meier \& Turner 2004). In NGC 1614, the gas with the highest $\mathcal{R}_{10}$ ratios is located in the dust lane, away from the bulk of the ongoing star formation. This most likely excludes the notion of high gas temperatures as the cause of the elevated line ratio. An alternative mechanism to elevate $\mathcal{R}_{10}$, and the absence of star formation in the dust lane, could be mechanical heating and shears caused by shocks. So far, no indications for shocks in the dust lane in NGC 1614 have been found: ${ }^{13} \mathrm{CO} 2-1$ observations at comparable spatial resolutions (Wilson et al. 2008) indicate an emission distribution similar to what we present for ${ }^{13} \mathrm{CO} 1-0$ in this work. Assuming that the elevated $\mathcal{R}_{10}$ values are due to the presence of high-density gas, we expect densities of about $3 \times 10^{3} \mathrm{~cm}^{-3}$ or more. The result would be that the ${ }^{13} \mathrm{CO} 2-1$ flux is a factor of four or more higher than for ${ }^{13} \mathrm{CO} 1-0$. This would have been picked up by the observations of Wilson et al. (2008). Thus, the presence of high-density gas due to shocks can most likely be ruled out as the determining factor for the high line ratios in NGC 1614. Furthermore, observations of dense gas tracers like $\mathrm{HCN}, \mathrm{HCO}^{+} 4-3$ and $\mathrm{CO}$ 6-5 (Imanishi \& Nakanishi 2013; Sliwa et al. 2014) have not yielded a detection of dense gas emission in the dust lane, their emission is solely found in the circumnuclear ring.

\subsubsection{Density}

Change in the gas density is a valid option to cause the observed change in $\mathcal{R}_{10}$ in NGC 1614 , however. A decrease in the gas density may cause an increase in the line ratio: if the gas in the dust lane is diffuse, that is, in the form of nonselfgravitating clouds, the lower critical density favors the emission of ${ }^{12} \mathrm{CO} 1-0$ photons. As a result of the so-called radiative trapping the ${ }^{12} \mathrm{CO}$ line is still bright at critical densities of $\sim 200$ $300 \mathrm{~cm}^{-3}$, where ${ }^{13} \mathrm{CO} 1-0$ is faint (Meier \& Turner 2004). Previous studies of nearby galaxies have suggested that the diffuse gas in the dust lane is due to the funneling of gas along the same, thus gas infall is involved (e.g., Aalto \& Hüttemeister 2000). If the infalling gas is the only effect causing the increase of $\mathcal{R}_{10}$, the abundance ratio over the central region would need to be well mixed, that is, it should have values more corresponding to what is found for the inner Galactic disk (Meier \& Turner 2004). $\mathcal{R}_{10}$ around 30 at the center of NGC 1614 places the line ratios for the molecular gas there firmly above this (see also Sect. 3.5; e.g., Aalto et al. 1995; Meier \& Turner 2004; Aalto et al. 2010).

One possible candidate for a secondary line ratio enhancement mechanism could be a density wave in NGC 1614. The situation in M 51 seems to be a good analogy for what we find in NGC 1614. In M 51, Tosaki et al. (2002) found high ${ }^{12} \mathrm{CO}-$ to- $^{-}$ ${ }^{13} \mathrm{CO} 1-0$ ratios in the central and interarm regions. Velocity dispersion observations led them to suggest the presence of streaming motions. The authors thus proposed that streaming motions, caused by density wave activity, led to the accumulation of dense gas located in self-gravitating clouds, as traced by ${ }^{13} \mathrm{CO} 1-0$, to be located downstream from the diffuse gas that is traced by ${ }^{12} \mathrm{CO} 1-0$. This is exactly what we find in NGC 1614: the $\mathcal{R}_{10}$ is high in the dust lane where we suspect the gas to be diffuse. Downstream of this gas, traced by ${ }^{12} \mathrm{CO} 1-0$, lies the peak of the ${ }^{13} \mathrm{CO} 1-0$ distribution. This is also similar to what has been proposed for the Medusa merger (Aalto et al. 2010).

\subsubsection{Abundance effects}

\subsubsection{1. ${ }^{12} \mathrm{C} /{ }^{13} \mathrm{C}$}

The ${ }^{12} \mathrm{CO}$-to- ${ }^{13} \mathrm{CO} 1-0$ intensity line ratio map shows values of about 30 toward the nucleus of NGC 1614 (see Sects. 3.5 and 4.1.1), which corresponds to line ratios found predominantly for warm, turbulent, high-pressure gas in the centers of luminous merging galaxies (e.g., Aalto et al. 1995; Glenn \& Hunter 2001). 



Fig. 7. Cartoon representation of the molecular gas structures in NGC 1614 (left) and an HST image of the same region for comparison (right). A high percentage of the ${ }^{12} \mathrm{CO} 1-0$ emission (hatched red) is associated with the dust lane. The gas in the dust lane is in the form of diffuse gas. The ${ }^{13} \mathrm{CO} 1-0$ emitting gas is not fully distributed throughout the dust lane (hatched gray). Its emission peak (blue star) is located in the "umbilical cord", the connection between the molecular gas in the circumnuclear ring, traced by ${ }^{12} \mathrm{CO} 2-1$ emission (crosshatch pattern, König et al. 2013), and the dust lane. The diffuse gas is being transported along the dust lane in the form of unbound molecular clouds (GMCs). The interaction with density waves (red arrows) causes the gas in the clouds to become self-gravitating. In the umbilical cord, crowding processes in the merger potential occur and the GMCs form GMAs through collisional coagulation. These larger associations are then transported further onto the circumnuclear ring and star formation may be triggered.

From the discussion of the excitation effects on the line ratios in Sect.4.1.1, we conclude that diffuse gas in the dust lane under the influence of density wave activity can partially explain elevated values for $\mathcal{R}_{10}$. To increase the ${ }^{12} \mathrm{CO}-$ to- $^{13} \mathrm{CO} 1-0$ to a level as we find in NGC 1614 an additional factor has to be taken into account to explain the enrichment of ${ }^{13} \mathrm{CO}$, which also causes the relative deficiency of $\mathrm{C}^{18} \mathrm{O}$. This is the effect of changing abundances.

Casoli et al. (1992) suggested that $\mathcal{R}_{10}$ could change depending on the ${ }^{12} \mathrm{CO} /{ }^{13} \mathrm{CO}$ abundance ratio, $\left[{ }^{12} \mathrm{CO}\right] /\left[{ }^{13} \mathrm{CO}\right]$, when low-metallicity gas was transported from the outskirts of a merger to its center. The starburst would then contribute to enrich the infalling metal-poor gas in ${ }^{13} \mathrm{C}$ (e.g., Rupke et al. 2008; Henkel et al. 2010). We assume the ${ }^{12} \mathrm{CO} 1-0$ line transition to be optically thick $(\tau=1)$, that the ${ }^{12} \mathrm{CO}$ and ${ }^{13} \mathrm{CO}$ emission trace the same gas and also that the excitation temperatures for the two are comparable ${ }^{3}$. This would mean that $\left[{ }^{12} \mathrm{CO}\right] /\left[{ }^{13} \mathrm{CO}\right]$ would be roughly 90 , which is a typical value for gas farther out in the Galactic disk (e.g., Henkel et al. 1985, 2014; Wilson \& Rood 1994; Wouterloot \& Brand 1996), and thus points to the presence of infalling, chemically less processed gas.

\subsubsection{2. ${ }^{16} \mathrm{O} /{ }^{18} \mathrm{O}$}

Using the $\mathrm{C}^{18} \mathrm{O} 1-0$ integrated intensity upper limit in the central $4^{\prime \prime} \times 4^{\prime \prime}$, the ${ }^{12} \mathrm{C}^{16} \mathrm{O}$-to- ${ }^{12} \mathrm{C}^{18} \mathrm{O} 1-0$ line ratio results in a lower limit of $\sim 325$. The resulting abundance ratio is $\left[{ }^{12} \mathrm{CO}\right] /\left[\mathrm{C}^{18} \mathrm{O}\right] \sim 900$. Values found for the Galactic center are $\sim 250$, for the solar neighborhood typical values are about 500 (e.g. Wilson \& Matteucci 1992). The $\left[{ }^{12} \mathrm{CO}\right] /\left[\mathrm{C}^{18} \mathrm{O}\right]$ ratio is significantly higher in NGC 1614 than in the LIRG $\mathrm{Zw} 049.057$ (Falstad et al. 2015), but comparable to what has been found for another LIRG, NGC 4418, (González-Alfonso et al. 2012). It is also comparable to values found in the merger Arp 299 (Falstad et al., in prep.). Interestingly, both Arp 299 and NGC 4418 have molecular gas

\footnotetext{
3 For more details see Appendix B.
}

inflows (Falstad et al. in prep., Costagliola et al. 2013) - Arp 299 also hosts an efficient starburst (e.g., Bondi et al. 2012, and references therein) - just like NGC 1614. Considering that this result for NGC 1614 is only a lower limit to $\left[{ }^{12} \mathrm{CO}\right] /\left[\mathrm{C}^{18} \mathrm{O}\right]$, and also taking the high ${ }^{13} \mathrm{CO}$-to- $\mathrm{C}^{18} \mathrm{O} 1-0$ integrated intensity line ratio in the central $4^{\prime \prime} \times 4^{\prime \prime}$ into account $(\sim 10.3)$, $\mathrm{C}^{18} \mathrm{O} 1-0$ seems very deficient in comparison to ${ }^{12} \mathrm{CO} 1-0$, but especially with respect to ${ }^{13} \mathrm{CO} 1-0 . \mathrm{C}^{18} \mathrm{O}$ is thought to come from short-lived massive stars early during a starburst event (e.g., Prantzos et al. 1996; Meier \& Turner 2004), whereas ${ }^{13} \mathrm{CO} 1-0$ is predicted to be produced later on in intermediate-mass stars (e.g., Meier \& Turner 2004, and references therein). An explanation for this deficiency in the center of NGC 1614 could be that the mixing of the infalling, chemically unprocessed gas with the prevailing gas is not efficient. Although this could also be an effect of the size of the beam in the observations - if the unprocessed infalling gas is located inside the same beam as the prevailing nuclear gas a dilution of the separate signals could occur. This dilemma will be solved by higher resolution observations of the same set of molecular tracers.

Taking the findings in Sect.4.1.1 and this section into account, we propose that the increase in the ${ }^{12} \mathrm{CO}-$ to- $^{13} \mathrm{CO}$ ratio in NGC 1614 is caused by the diffuse gas in the dust lane and density wave activity, in combination with enrichment of ${ }^{13} \mathrm{CO}$ due to infalling metal-poor gas from farther out in the galactic disk. However, a definitive answer to whether a change in the temperature of the gas also has an influence can only be obtained together with sensitivity-matched observations of the ${ }^{12} \mathrm{CO}$ and ${ }^{13} \mathrm{CO} 2-1$ lines.

\subsection{NGC 1614 - the big picture?}

In this section, we now collocate our results together with what has been previously reported on what is going on in NGC 1614. A cartoon representation of the proposed scenario is depicted in Fig. 7. 
In 2001, a starburst ring was discovered at the nucleus of NGC 1614 by Alonso-Herrero et al. Its presence was confirmed in a number of other tracers (e.g., Olsson et al. 2010; Väisänen et al. 2012). Alonso-Herrero et al. (2001) suggested that the ring was formed as the result of an outward progressing starburst event that has already consumed most of the gas at its center. The ring also has a molecular component (König et al. 2013; Sliwa et al. 2014; Xu et al. 2015). As a result, it was suggested that the ring is situated at the location of crowded orbits in the merger potential where it is replenished by gas coming in along the dust lane (König et al. 2013; Sliwa et al. 2014). The ${ }^{12}$ CO $1-0$ (Olsson et al. 2010; Sliwa et al. 2014; García-Burillo et al. 2015), 2-1 (König et al. 2013) and ${ }^{13} \mathrm{CO} 1-0$ line transitions trace the low-to-intermediate surface brightness gas that shows how the starburst ring is connected to the large-scale molecular gas reservoir: diffuse gas in the form of unbound giant molecular clouds (GMCs) could be funneled along the dust lane from the molecular gas reservoir at larger scales (seen in ${ }^{12} \mathrm{CO} 1-0$ ) toward the nucleus. During this process, the gas may be hit by a density wave. The resulting shocks could cause the gas density to increase and the molecular clouds to become self-gravitating (as traced by ${ }^{13} \mathrm{CO} 1-0$ ). In this scenario, the molecular clouds are trapped at the connection between the dust lane and the ring, "the umbilical cord" (described by König et al. 2013), inside the mergers potential through crowding processes, for instance. This could lead to collisional coagulation of the GMCs into larger-sized giant molecular associations (GMAs, traced by, e.g., $\left.{ }^{12} \mathrm{CO} 2-1\right)$ that then possibly migrate onto the circumnuclear ring. In this way, the nuclear gas reservoir can be constantly replenished. The cloud-cloud interactions could also trigger the onset of star formation in the ring (Scoville et al. 1986; Tan 2000): the high-density gas, such as $\mathrm{HCN}$ and $\mathrm{HCO}^{+}$(e.g., Imanishi \& Nakanishi 2013; Sliwa et al. 2014; Xu et al. 2015), is exclusively associated with the star formation itself.

Whether the ring is indeed caused by a wildfire expanding into the surrounding molecular medium or if is solely formed at the location of the resonance in the merger potential is still under discussion. Solving this argument will require further studies: resolution- and sensitivity-matched ${ }^{12} \mathrm{CO}$ and ${ }^{13} \mathrm{CO} 2-1$ observations will greatly improve our ability to determine whether the presence of non-selfgravitating gas in the dust lane or a change in the temperature of the gas is the cause of the extreme line ratios in NGC 1614. Higher resolution ${ }^{12} \mathrm{CO} 1-0$ observations at high sensitivity are needed to search for indicators of possible streaming motions between the large-scale and small-scale molecular gas reservoirs to verify the funneling of gas along the dust lanes toward the circumnuclear ring. They would also allow us to study the possible connection between the $\mathrm{CO} 1-0$ and the CO 2-1 gas reservoirs in more detail, thus allowing to collect evidence in favor or against the competing scenarios proposed for the formation of the circumnuclear ring.

We would also like to stress that our results emphasize the need to be cautious which tracers of the molecular gas to best use to determine star formation capabilities, also for studies at high redshifts. We have especially shown in this paper that the ${ }^{12} \mathrm{CO} 1-0$ emission is not necessarily a tracer of the overall content of the molecular gas that is capable to partake in star formation because a high percentage of it might be in the form of diffuse molecular gas. Additional observations at higher- $J \mathrm{CO}$ transitions are necessary to conclude on this. Furthermore, $\mathrm{C}^{18} \mathrm{O}$ should be used with caution to determine the age of the starburst activity in galaxies with proven gas infall. The mixing of the infalling gas with the prevailing gaseous materials might falsify the age determination to a large degree.

\section{Summary}

In summary, with the ALMA observations presented here, we showed that the nearby starburst galaxy NGC 1614 harbors large reservoirs of molecular gas traced by ${ }^{12} \mathrm{CO}$ and ${ }^{13} \mathrm{CO} 1-0$. The ${ }^{12} \mathrm{CO}$ emission is widely distributed throughout the galaxy and has a strong connection to the dust lanes, whereas the ${ }^{13} \mathrm{CO}$ emission is much more compact and seems to avoid them. This possibly indicates non-selfgravitating, diffuse gas in the dust lane that originates from farther out in the galaxy disk. The ${ }^{13} \mathrm{CO} 1-0$ emission distribution is most likely a result of the effect of the progression of density waves on the galaxies molecular medium. In addition to ${ }^{12} \mathrm{CO}$ and ${ }^{13} \mathrm{CO} 1-0$, other molecular gas tracers such as $\mathrm{CN} 1-0$ and $\mathrm{CS} 2-1$ are present in NGC 1614 . For $\mathrm{C}^{18} \mathrm{O}$ only an upper limit was found.

Acknowledgements. This paper makes use of the following ALMA data: ADS/JAO.ALMA\#2013.1.00991.S. ALMA is a partnership of ESO (representing its member states), NSF (USA) and NINS (Japan), together with NRC (Canada), NSC and ASIAA (Taiwan), and KASI (Republic of Korea), in cooperation with the Republic of Chile. The Joint ALMA Observatory is operated by ESO, AUI/NRAO and NAOJ. This research has made use of the NASA/IPAC Extragalactic Database (NED) which is operated by the Jet Propulsion Laboratory, California Institute of Technology, under contract with the National Aeronautics and Space Administration.

\section{References}

Aalto, S., \& Hüttemeister, S. 2000, A\&A, 362, 42

Aalto, S., Booth, R. S., Black, J. H., \& Johansson, L. E. B. 1995, A\&A, 300, 369

Aalto, S., Radford, S. J. E., Scoville, N. Z., \& Sargent, A. I. 1997, ApJ, 475, L107

Aalto, S., Beswick, R., \& Jütte, E. 2010, A\&A, 522, A59

Alonso-Herrero, A., Engelbracht, C. W., Rieke, M. J., Rieke, G. H., \& Quillen, A. C. 2001, ApJ, 546, 952

Bondi, M., Pérez-Torres, M. A., Herrero-Illana, R., \& Alberdi, A. 2012, A\&A, 539, A134

Bournaud, F., Jog, C. J., \& Combes, F. 2005, A\&A, 437, 69

Casoli, F., Dupraz, C., \& Combes, F. 1992, A\&A, 264, 55

Combes, F. 1988, in NATO ASIC Proc. 232: Galactic and Extragalactic Star Formation, eds. R. E. Pudritz, \& M. Fich, 475

Costagliola, F., Aalto, S., Rodriguez, M. I., et al. 2011, A\&A, 528, A30

Costagliola, F., Aalto, S., Sakamoto, K., et al. 2013, A\&A, 556, A66

Dahmen, G., Huttemeister, S., Wilson, T. L., \& Mauersberger, R. 1998, A\&A, 331,959

Davis, T. A., Rowlands, K., Allison, J. R., et al. 2015, MNRAS, 449, 3503

Downes, D., \& Solomon, P. M. 1998, ApJ, 507, 615

Falstad, N., González-Alfonso, E., Aalto, S., et al. 2015, A\&A, 580, A52

García-Burillo, S., Combes, F., Usero, A., et al. 2015, A\&A, 580, A35

Glenn, J., \& Hunter, T. R. 2001, ApJS, 135, 177

González-Alfonso, E., Fischer, J., Graciá-Carpio, J., et al. 2012, A\&A, 541, A4 González-Alfonso, E., Fischer, J., Graciá-Carpio, J., et al. 2014, A\&A, 561, A27

Henkel, C., \& Mauersberger, R. 1993, A\&A, 274, 730

Henkel, C., Guesten, R., \& Gardner, F. F. 1985, A\&A, 143, 148

Henkel, C., Downes, D., Weiß, A., Riechers, D., \& Walter, F. 2010, A\&A, 516, A111

Henkel, C., Asiri, H., Ao, Y., et al. 2014, A\&A, 565, A3

Imanishi, M., \& Nakanishi, K. 2013, AJ, 146, 47

Israel, F. P. 2009, A\&A, 493, 525

Jogee, S. 2006, in Physics of Active Galactic Nuclei at all Scales, ed. D. Alloin (Berlin: Springer Verlag), Lecture Notes in Physics, 693, 143

König, S., Aalto, S., Muller, S., Beswick, R. J., \& Gallagher, J. S. 2013, A\&A, 553, A72

König, S., Aalto, S., Lindroos, L., et al. 2014, A\&A, 569, A6

Kotilainen, J. K., Reunanen, J., Laine, S., \& Ryder, S. D. 2001, A\&A, 366, 439 


\section{S. König et al.: Deep ALMA imaging of NGC 1614}

McMullin, J. P., Waters, B., Schiebel, D., Young, W., \& Golap, K. 2007, in Astronomical Data Analysis Software and Systems XVI, eds. R. A. Shaw, F. Hill, \& D. J. Bell, ASP Conf. Ser., 376, 127

Meier, D. S., \& Turner, J. L. 2004, AJ, 127, 2069

Naab, T., Johansson, P. H., \& Ostriker, J. P. 2009, ApJ, 699, L178

Olsson, E., Aalto, S., Thomasson, M., \& Beswick, R. 2010, A\&A, 513, A11

Polk, K. S., Knapp, G. R., Stark, A. A., \& Wilson, R. W. 1988, ApJ, 332, 432

Prantzos, N., Aubert, O., \& Audouze, J. 1996, A\&A, 309, 760

Puxley, P. J., \& Brand, P. W. J. L. 1999, ApJ, 514, 675

Rupke, D. S. N., Veilleux, S., \& Baker, A. J. 2008, ApJ, 674, 172

Sanders, D. B., Mazzarella, J. M., Kim, D.-C., Surace, J. A., \& Soifer, B. T. 2003, AJ, 126, 1607

Schwartz, C. M., \& Martin, C. L. 2004, ApJ, 610, 201

Scoville, N. Z., Soifer, B. T., Neugebauer, G., et al. 1985, ApJ, 289, 129

Scoville, N. Z., Sanders, D. B., \& Clemens, D. P. 1986, ApJ, 310, L77

Scoville, N. Z., Sanders, D. B., Sargent, A. I., Soifer, B. T., \& Tinney, C. G. 1989, ApJ, 345, L25

Shlosman, I., Frank, J., \& Begelman, M. C. 1989, Nature, 338, 45
Simkin, S. M., Su, H. J., \& Schwarz, M. P. 1980, ApJ, 237, 404

Sliwa, K., Wilson, C. D., Iono, D., Peck, A., \& Matsushita, S. 2014, ApJ, 796, L15

Tan, J. C. 2000, ApJ, 536, 173

Taniguchi, Y., Ohyama, Y., \& Sanders, D. B. 1999, ApJ, 522, 214

Tosaki, T., Hasegawa, T., Shioya, Y., Kuno, N., \& Matsushita, S. 2002, PASJ, 54, 209

Väisänen, P., Rajpaul, V., Zijlstra, A. A., Reunanen, J., \& Kotilainen, J. 2012, MNRAS, 420, 2209

van Dishoeck, E. F., \& Black, J. H. 1988, ApJ, 334, 771

Visser, R., van Dishoeck, E. F., \& Black, J. H. 2009, A\&A, 503, 323

Weinzirl, T., Jogee, S., Khochfar, S., Burkert, A., \& Kormendy, J. 2009, ApJ, 696, 411

Wilson, T. L., \& Matteucci, F. 1992, A\&ARv, 4, 1

Wilson, T. L., \& Rood, R. 1994, ARA\&A, 32, 191

Wilson, C. D., Petitpas, G. R., Iono, D., et al. 2008, ApJS, 178, 189

Wouterloot, J. G. A., \& Brand, J. 1996, A\&AS, 119, 439

Xu, C. K., Cao, C., Lu, N., et al. 2015, ApJ, 799, 11 


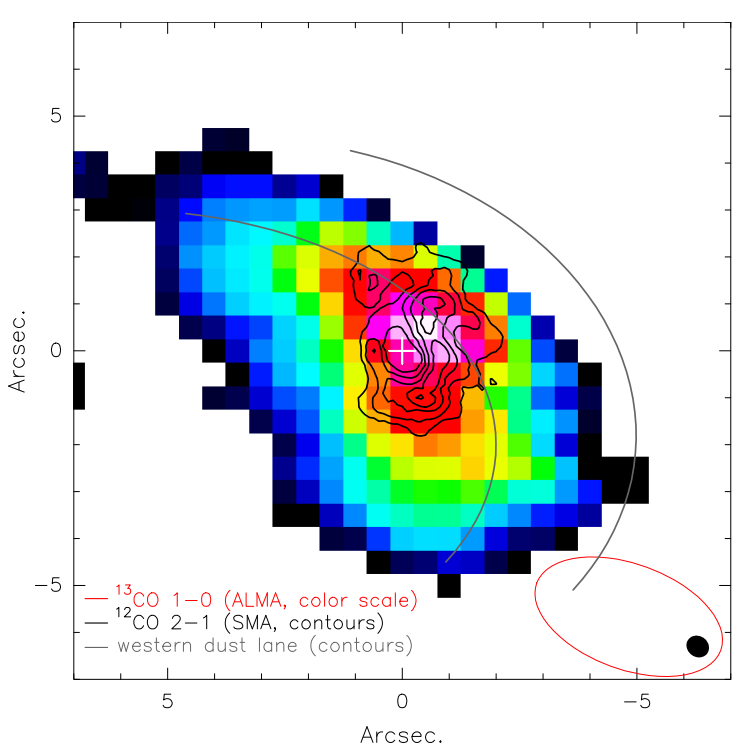

Fig. A.1. Comparison of high-resolution ${ }^{12} \mathrm{CO} 2-1$ observations with the SMA (black contours, König et al. 2013) to the ALMA ${ }^{13} \mathrm{CO} 1-0$ emission presented in this work (color scale). Note how the ${ }^{13} \mathrm{CO} 1-0$ emission peaks exactly at the location of the connection between the molecular ring and the dust lane (grey contours, see also Figs. 1a \& 3 in König et al. 2013). The ${ }^{12} \mathrm{CO} 2-1$ contours start at $4 \sigma$ and are spaced in steps of $4 \sigma\left(1 \sigma=6.16 \mathrm{mJy} \mathrm{beam}^{-1}\right)$. North is up, east to the left, and the beams are shown in the lower right corner of the image. The cross marks the position of the phase center of the observations.

\section{Appendix A: Comparison overlays of ${ }^{12} \mathrm{CO} 1-0,2-1$ and ${ }^{13} \mathrm{CO} 1-0$ emission distributions}

Figures A.1 and A.2 show overlays of ${ }^{12} \mathrm{CO} 1-0,2-1$, and ${ }^{13} \mathrm{CO} 1-0$ that might be useful for a closer look at the detailed structure of NGC 1614. The observations used here are presented in this work or have been previously published.

\section{Appendix B: Conversion of line ratios into abundance ratios}

To study the effects of changes in abundance ratios vs. the effects of different excitation mechanisms, we used the ${ }^{12} \mathrm{CO}$ to- ${ }^{13} \mathrm{CO} 1-0$ ratio distribution in NGC 1614 to derive the abundance ratio $\left(\left[{ }^{12} \mathrm{CO}\right] /\left[{ }^{13} \mathrm{CO}\right]\right)$. While $\tau\left({ }^{12} \mathrm{CO} 1-0\right)$ in galaxy disks (i.e., an ensemble of GMCs) may well exceed unity, there is mounting evidence that the emitting $\mathrm{CO}$ surfaces in galaxy centers have moderate optical depths (e.g., Aalto et al. 1995; Downes \& Solomon 1998; Israel 2009), this is also true for the Galactic center (e.g., Polk et al. 1988; Dahmen et al. 1998). Since a $\tau=1$ surface would have the optical combination of filling factor and brightness temperature it is a reasonable assumption that much of the $\mathrm{CO}$ emission would be emerging from these surfaces. Thus, we use as a first-order approximation of the optical depth of $\tau=1$ in our data as a starting point to study the

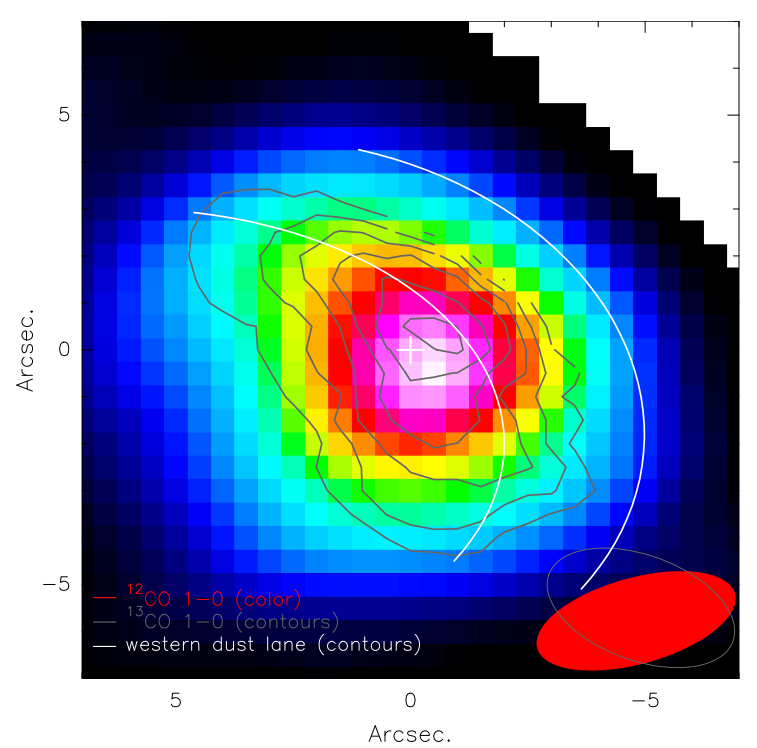

Fig. A.2. Overlay of the ${ }^{13} \mathrm{CO} 1-0$ emission observed with ALMA (gray contours) on top of the ${ }^{12} \mathrm{CO} 1-0$ emission observed with ALMA (color scale). Note how the bulk of the ${ }^{13} \mathrm{CO} 1-0$ emission is located outside the dust lane to the west of the nucleus (white contours). Moreover, the ${ }^{13} \mathrm{CO} 1-0$ located in the dust lane does not cover its full extent. The ${ }^{13} \mathrm{CO} 1-0$ contours are the same as in Fig. $3 \mathrm{~b}$. North is up, east to the left, and the beams are shown in the lower right corner of the image. The cross marks the position of the phase center of the observations.

physical gas properties and mechanisms with a simple model. This is justified for the molecular gas in NGC 1614 based on several pieces of evidence: 1) In the dust lane the gas density is low: Studies associate high-density gas tracers exclusively with the circumnuclear ring (e.g., Imanishi \& Nakanishi 2013; Sliwa et al. 2014; Xu et al. 2015); thus, the brightness temperature measured in the dust lane suggests that the gas has a $\tau$ of $\geq 1$. If the emission is optically thin in a cool, low-density environment, its brightness temperature is $<1 \mathrm{~K}$ at this resolution. 2) In the dense and warmer gas in the center (e.g., Imanishi \& Nakanishi 2013; Sliwa et al. 2014; Xu et al. 2015), even optically thin ${ }^{12} \mathrm{CO} 1-0$ may have $T_{\mathrm{B}}<1 \mathrm{~K}$. However, the $\tau \geq 1$ surfaces would contribute more to the total intensity and ${ }^{12} \mathrm{CO}$ is bathed in an intense field in the starburst region and cannot self-shield until $\tau=1$ (e.g., van Dishoeck \& Black 1988; Visser et al. 2009). The high ${ }^{13} \mathrm{CO}$-to- $\mathrm{C}^{18} \mathrm{O}$ ratio supports this. In NGC 1614, the gas with the highest $\mathcal{R}_{10}$ ratios is located in the dust lane, away from the bulk of the ongoing star formation, and thus away from the most intense radiation. Therefore, the intense radiation field from the star formation in the ring and/or self-shielding of ${ }^{12} \mathrm{CO}$ are unlikely to cause the high $\mathcal{R}_{10}$ (and the high isotopic ratio) in the western dust lane.

More higher resolution studies of the CO 1-0 isotopologs are necessary to set a tighter constraint on the optical depth structure of the molecular gas in NGC 1614. 